\title{
Erster Schritt in Richtung bundesweiter MTRA-Ausbildungsvergütung
}

Im neu ausgehandelten Tarifvertrag zwischen ver.di und der Vereinigung der kommunalen Arbeitgeberverbände wurde vereinbart, dass MTRA-Auszubildende ab dem 01. Januar 2019 eine Ausbildungsvergütung erhalten. Davon profitieren aber zunächst nicht alle MTRA-Schülerinnen und Schüler, sondern nur jene, die einen Ausbildungsvertrag bzw. ein Ausbildungsverhältnis mit einem kommunalen bzw. Universitätskrankenhaus haben.

Sind die Vorrausetzungen erfüllt, gibt es im ersten Ausbildungsjahr 965,24€ pro Monat, im zweiten Ausbildungsjahr 1.025,30€ pro Monat und ab dem dritten Ausbildungsjahr $1.122,03 €$ pro Monat. Hinzu kommen Urlaubsansprüche, Jahressonderzahlungen und Freistellungen für die Prüfungsvorbereitungen. Unklar ist, wie die Urlaubstage und Freistellungen mit den bisherigen Ferienzeiten verrechnet werden. MTRA wurden bislang schulisch ausgebildet und das war mit den üblichen Ferienzeiten (ca. 3 Monate im Jahr) verbunden.

Bevor der Tarifvertrag verbindlich wird, muss aber noch die Erklärungsfrist am
14. Dezember 2018 abgewartet werden. Der Tarifvertrag ist bisher noch nicht veröffentlicht. Das ist aber notwendig, damit die VMTB die Tarifeinigung und deren Umsetzung abschließend beurteilen kann.

Die VMTB begrüßt diesen ersten Schritt zur bundesweiten Vergütung der MTRA-Ausbildung. Mittelfristig müssen aber alle MTRASchülerinnen und -Schüler davon profitieren und zwar unabhängig davon, wo sie ausgebildet werden. 